\title{
AOMDV Protocol: A Literature Review
}

\author{
Bhawna Mathur, Anuj Jain
}

\begin{abstract}
MANET has received the significant attention in every field of Information and Communication Technology industry. The nodes in Mobile Ad hoc networks continuously move leading to randomly changing topology which further leads to many problems such as link breakages and loss of packets sent by the source node to the destination. In MANETs, the nodes are mobile and battery operated. As the nodes have limited battery resources and multi hop routes are used over a changing network environment due to node mobility, it requires energy efficient routing protocols to limit the power consumption, prolong the battery life and to improve the robustness of the system. This paper evaluates the performance of various adhoc routing protocols such as DSDV, AODV, DSR, TORA and AOMDV in terms of energy efficiency and it also proposes a new routing algorithm that modifies AOMDV and it provides better performance compared to all the above protocols. This paper gives an overview of the MANET and AOMDV protocol .It also explains its advantages and disadvantages.
\end{abstract}

Index Terms - AODV,AOMDV,Congestion,MAODV,QOS.

\section{INTRODUCTION}

A Mobile Ad hoc Network is an autonomous collection of mobile nodes and communicated through wireless links. It is a temporary network without having any centralized access point, infrastructure, or centralized administration.

The basic architecture of MANET consists of nodes that are dynamically self-organized into arbitrary and temporary network topology without any infra structure support It is an wireless network that perform multi hop communication between mobile nodes, without the reliance on a fixed base station. Each node acts as router. A routing protocol is needed while a packet is needed to be transmitted to a destination [1].

The advantage of employing MANET is to offer a large degree of freedom at a minimal cost in comparison to other networking solutions. The ease and speed of deployment of these networks make them ideal for recovery after a natural or manmade disaster, business associates sharing information during a meeting or conference, and military communications in a battlefield.

\section{A. Characteristics of MANET}

MANET possesses the characteristics of wireless network in general, and extra aspects that are particular to the Ad Hoc Networking are -:

- Infrastructure-free: MANET is a self-organized network. It is independent of any established infrastructure and centralized network administration. India

Bhawna Mathur, E.C.E Department, Bhagwant University, Ajmer,

Dr. Anuj Jain, E.C.E Department, Bhagwant University, Ajmer, India,
Here each individual node plays as a router and operates in distributed manner.

- Multi-hop routing: Due to the absence of any dedicated router, here every node acts as a router and aids in forwarding packets to the destination node. That's how information sharing among mobile nodes is made available.

- Dynamic network topology: The topology of MANET changes frequently as MANET nodes move randomly in the network, hence leading to regular route changes, network partitions, and possibly packet losses.

- Variation on link and node capabilities: Every participating node in an ad hoc network is constituted with distinctive type of radio devices having varying transmission and receiving capabilities. They all operate on multiple frequency bands. Due to this heterogeneity in the radio capabilities, asymmetric links may be formed.

- Energy-constrained working: Due to the limited power supply of the batteries carried by portable mobile devices, the processing power of node is restricted.

- Scalable Network: Numerous network management algorithms have been designed to work on fixed or relatively small wireless networks. A wide range of MANET applications may involve bulky networks with plenty of nodes especially that can be found in strategic networks. Scalability is critical to the advantageous operation of MANET.

\section{B. Introduction to Routing Protocols in MANETs}

Routing is the process of interchange information from one program to the other program in a network.

a) Proactive Routing Protocols

This protocol is also known as a table driven protocol and each node maintains a routing table will consists of the information of routing to every node in the network. Since the nodes are mobile, they keep on the changing their location. It maintained the routing tables which are periodic or whenever a change occurs, are updated provide a number of proactive routing protocols [10].

b) Reactive Routing Protocol

This protocol is also known as the on-demand routing protocol which they do not maintain the routing information or activity

of routing at the network nodes, which there is no communication [10].

c) Hybrid Routing Protocol

This is the combination of the proactive and reactive protocols which work well for the networks with a small 
number of nodes. While the hybrid reactive/proactive protocols are used to achieve the high performance as the number of nodes increases [10]. It is a key of idea to use a reactive routing at the global network level while the employing a proactive in a node's local neighborhood. The examples of hybrid routing protocol are: ZRP (Zone Routing Protocol) and ZHLS (Zone-based Hierarchical Link State).

\section{AOMDV (ADHOC ON DEMAND MUltipath Distance VECTOR)}

It is a reactive routing protocol, search path when required. AOMDV is a multi-path routing protocol. It is an extension to AODV and also provides two main services i.e. route discovery and maintenance.

AOMDV routing Protocol is On-demand routing protocols for MANETs discover a route when a source needs to communicate with a destination. The multi-path routing protocol discovers multiple paths during the single route discovery process. These multiple paths can be used for load spreading or as backup routes when the primary route fails. Like AODV, AOMDV is based on distance vector concept and uses hop by hop routing approach. Moreover, AOMDV also finds routes on demand using a route discovery procedure. Unlike AODV, AOMDV finds multiple routes in a single route discovery procedure. In AODV all duplicate RREQs are discarded whereas AOMDV look for an opportunity of getting an alternate route with each duplicate RREQ. In AOMDV, RREQ propagation from the source towards the destination establishes multiple reverse paths both at intermediate nodes as well as the destination. Multiple RREPs traverse these reverse paths back, to form multiple forward paths to the destination at the source and intermediate nodes. The core of the AOMDV protocol lies in ensuring that multiple paths discovered are loop free and disjoint; and in efficiently finding such paths using a flood-based route discovery. AOMDV route update rules, applied locally at each node, play a key role in maintaining loop-freedom and disjoint properties [19].

Being the hop-by-hop routing protocol, the intermediate node can maintain multiple path entries in their respective routing table. To discover distinct paths, AOMDV suppresses duplicate route requests (RREQs) at intermediate nodes. Such suppression comes in two different variations, resulting in either node (illustrated in Fig. 1 (a)) or link (illustrated in Fig. 1(b)) disjoint. AOMDV can be configured to either discover the link (no common link between any given pair of nodes) or node (in addition to link disjoint, common intermediate nodes are also excluded between any given pair of nodes) disjoints paths. Disjoint alternate paths are a good choice than overlapping alternate paths, as the probability of their interrelated and concurrent failure is smaller. This property can be helpful in an adversarial environment where malicious activity can also cause additional link failure [12].

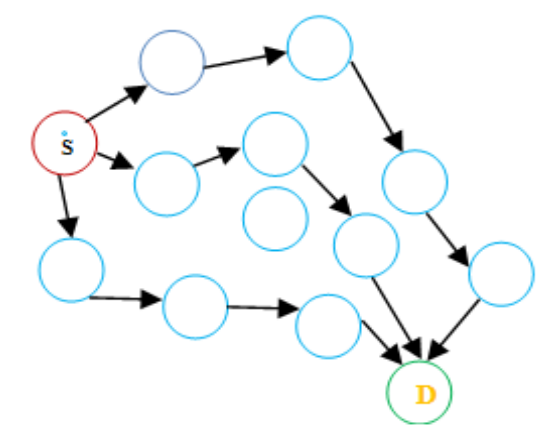

Fig 1 (a) Node Disjoint

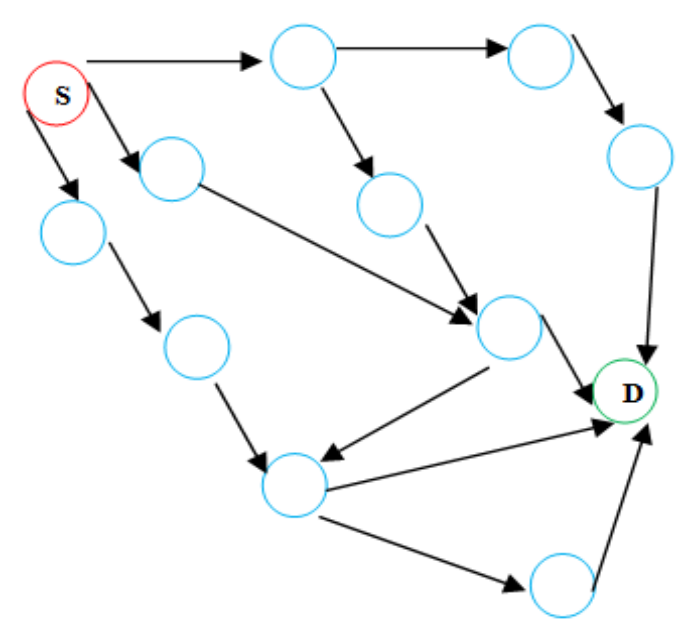

Fig 1 (b) Link Disjoint

Advantages of AOMDV-

1 AOMDV is more efficient.

2 It has less overhead.

3 AOMDV works more efficiently in dense and heavy networks.

Disadvantages of AOMDV-

1 Various malicious attacks hamper AOMDV. Black hole and gray holes attacks are the most important security issues in MANET.

\section{REVIEW OF WORK}

Due to the inherent design disadvantages of routing protocol in MANETs, many researchers have conducted diverse techniques to propose different types of prevention mechanisms for solving the problem. They have carried out numerous experiments and observations to illuminate the darkness of this field. Their findings and suggestions are reviewed here. Some of the papers have been considered that has been taken as a motivation towards my study.

1. B.Malarkodi, P.Rakesh and B.Venkataraman[3] evaluates the performance of AOMDV and AODV routing protocol in the presence of poisson and bursty self similar traffic and compares them with that of CBR traffic. Their simulation results indicate that the packet delivery fraction and throughput in AOMDV is increased in the presence of self similar traffic compared to other traffic. Further, the 
average end to end delay variation of AOMDV in all three cases of traffic models is almost same.

2. Shalini Puri and Dr. Satish.R.Devene [4]concentrate on Congestion Avoidance and load balancing in the network. The proposed protocol makes following changes in existing AOMDV protocol:

-Higher hop count routes are preserved in routing table.

- Paths are selected according to queue length and hop count.

- If queue length is large then alternate path is selected.

- Queue length decides RREQ packets are forward or discarded.

The proposed protocol avoids congestion, balance the load and to an extent avoid link failure. AODV- Multipath has improved packet delivery ratio, throughput, reduced packet delay and packet drop.

3. Salwa Othmen, Aymen Belghith, Faouzi Zarai, Mohammad S. Obaidat et al.[5] discussed the MANET dynamic topology and the limited battery power of mobile nodes. The power is a major constraint in ad hoc networks since the nodes operate with limited battery life. Hence, the author mainly consider power aware as a primary objective. Also, the support of QoS requirements in terms of delay and bandwidth becomes a challenge .The proposed protocol PDMRP has been evaluated with NS2 simulator. They show through simulation that their proposed routing protocol called Power and Delay aware Multi-path Routing Protocol (PDMRP) outperforms the Stable Path Routing Protocol based on Power Awareness (SPR) and the Modified Adhoc On-Demand Distance Vector (MAODV) routing protocols in terms of throughput, end-to-end delay, and loss rate.

Moreover, this protocol takes into account the life time as well as the number of path hopes in order to cleverly share energy consumption between stable nodes(having higher battery life).This routing protocol keeps backup paths in order to rapidly switching to operational routes when problems occur.

4.In V.Lalitha and Dr.R.S.Rajesh[6] proposed routing algorithmthe number of hops in a route are minimized,the overall network overhead is minimized and the overall energy consumption of the network is also minimized. They call this model asAOMDV_RR Range Routing and implemented it under ns2 by improving the standard AOMDV protocol. They studied the proposed AOMDV_RR and the standard AOMDV under different network densities and measured the performance for suitable metrics. Measurable difference in performance was realized and the proposed AOMDV_RR performed better than normal AOMDV with respect to metrics network overhead, throughput and energy consumption. Their arrived results proves the significant improvement in performance and reduction in overheads .

5. Gayatree Rana,Bikram Ballav and Dr. Binod Kumar Pattanayak[7] compared characteristics and behaviour of few adhoc communicating protocols namely Ad Hoc on demand Distance Vector (AODV) Ad hoc On-demand Multipath Distance vector(AOMDV), Dynamic Source Routing(DSR), Destination Sequence Distance Vector(DSDV) and Power
Aware Ad-hoc On demand Distance Vector (PAAODV).The performance of above routing protocol is compared for following performance characteristics i.e. throughput, energy conservation, ratio of packet delivery and average delay with respect to number of nodes. These simulation is performed using network simulator (NS2.35)

The results are as follows, AODV gives better performance as compared to other protocols in terms of throughput, PAAODV gives a better performance based on residual energy and DSR gives more packet delivery ratio and shortest end to end delay.

6. Abrar Omar Alkhamisi and Seyed M Buhari[8] proposed a Trust based Secured AOMDV to enhance the security of the network. This paper extends an Ad hoc On-Demand Multipath Distance Vector (AOMDV) Routing protocol, named as Trust-based Secured Ad hoc On-demand Multipath Distance Vector (TS-AOMDV), which is based on the nodes' routing behavior.

The proposed TSAOMDV aims at identifying and isolating the attacks such as flooding, black hole, and gray hole attacks in MANET. With the help of Intrusion Detection System (IDS) and trust-based routing, attack identification and isolation are carried out in two phases of routing such as route discovery and data forwarding phase. IDS facilitates complete routing security by observing both control packets and data packets that are involved in the route identification and the data forwarding phases. An IDS monitors the packet generation rate of the source and assigns an inversely proportional value of the request packet count as the 'source-trust value' of the corresponding source. When the trust value of the source reaches the threshold, the route request packet from the attacker source is dropped. In data forwarding phase, an attacker that involves as a router drops the packet instead of forwarding. The IDS monitors the packet forwarding activity of the router and assigns the 'router trust value' as the ratio between the forwarded packet count and the received packet count. When the trust value of the router reaches the threshold, it selects an alternate path from the multiple available paths that are stored in the routing table and resumes the data transmission through it. To improve the routing performance, the IDS integrates the measured statistics into the AOMDV routing protocol for the detection of attackers. Finally, AOMDV and TS-AOMDV is compared through the NS2 based simulation model. The performance evaluation reveals that the proposed TS-AOMDV improves the performance in terms of throughput by $57.1 \%$ more than that of an AOMDV under adversary scenario. The simulated results show the superiority of the proposed protocol in various scenarios.

7. Versha Matre and Reena Karandikar [9] describe that to get the Quality of Service (QoS) in MANET, Trust based Ad-Hoc On Demand Distance Vector (AODV) and Modified Ad-Hoc On-Demand Multicast Distance Vector (MAOMDV) routing protocols are analyzed. In this work author build some assumption and establish the network model of MAOMDV. In the MAOMDV, they additionally assume that the system is provided with some monitor mechanisms or intrusion detection units either within the network layer or the application layer so one node will 
observe the behaviours of its one-hop neighbours. Another kind of secure routing protocol that uses cryptography technologies. NS-2 is chosen as the simulation software for this study. It is concluded that the MAOMDV is best suited for designing a good quality oriented protocol for better throughput, Packet delivery ratio, Residual energy and larger coverage area with lower delay in multi hop.

\section{RESULT}

In this paper we have studied the various aspects of AOMDV protocol. We observe that AOMDV offers a significant reduction in delay. In general, AOMDV always offers a superior overall routing performance than AODV and other routing protocol in a variety of mobility and traffic conditions. We are currently working on efficient AOMDV protocol. After studying the various aspects of AOMDV we have concluded that AOMDV has many advantages but there are some shortcomings in AOMDV which needs to be improved.

Keeping in view the above findings, we conclude that the link disjoint path option of multi-path routing protocol overall performs better than single-path or node disjoint path option of multi-path routing protocol. . Results will be obtained as modified AOMDV providing better performance compared to AOMDV, AODV, TORA, DSR and DSDV protocols.

\section{REFERENCES}

[1] Er. Megha and Er. Tarun Bagga, “Performance Evaluation Of AOMDV Routing Protocol with Internet of Things" , Department of Computer Science, Haryana Engineering College, Jagadhri, Vol 5 Issue 3, May - June 2017.

[2] Behra Rajesh Umashankar, IIRakhi Kumari Purnima, “A Comparative Study of Topology and Position Based Routing Protocols in Mobile Ad Hoc Networks", Vol. 2, Issue 2, Ver. 1 (April - June 2014) ,IJARCST 2014.

[3] B.Malarkodi, P.Rakesh and B. Venkataramani, " Performance evaluation Of On-Demand Multipath Distance Vector Routing Protocol Under Different Traffic Models.”, International Conference On Advances in Recent Technologies in Communication And Computing 2009.

[4] Shalini Puri and Dr. Satish.R.Devene, "Congestion Avoidance and Load balancing in AODV- Multipath using Queue Length”, Second International Conference on Emerging Trends in Engineering And Technology, ICETET-2009.

[5] Salwa Othmen, Aymen Belghith, Faouzi Zarai and Mohammad S. Obaidat, "Power and Delay- aware Multi-path Routing Protocol for Ad Hoc Networks", IEEE - 2014.

[6] V.Lalitha and Dr.R.S.Rajesh, "Power Aware and Topology Aware Ad-Hoc On-Demand Multipath Distance Vector Routing For Manet ", IEEE -2014 .

[7] Gayatree Rana, Bikram Ballav and Dr. Binod Kumar Pattanayak, “ Performance analysis of Routing Protocols In Mobile Ad-hoc Network", International Conference on Information Technology 2015.

[8] Abrar Omar Alkhamisi and Seyed M Buhari, "Trusted Secure Adhoc On-Demand Multipath Distance Vector Routing in MANET", 30th International Conference on Advanced Information Networking and Applications 2016

[9] Versha Matre and Reena Karandikar, "Multipath Routing Protocol for Mobile Adhoc Networks", Symposium on CDAN 2016.

[10] Y.Zhang and W.Lee, "Intrusion Detection in Wireless Ad Hoc Networks", Proceedings of the 6th International Conference on Mobile Computing and Networking, Boston, Massachusetts, United States, 2000.
[11] Mahesh K. Marina and Samir R. Das, "On-demand Multipath Distance Vector Routing in Ad Hoc Networks" , Department of Electrical \& Computer Engineering and Computer Science University of Cincinnati.

[12] Abdur Rashid Sangi, Jianwei Liu, Zhiping Liu, "Performance Comparison of Single and Multi-Path Routing Protocol in MANET with Selfish Behaviours", World Academy of Science, Engineering and Technology 2010.

[13] G. Parissidis, V.Lenders, M.May and B.Plattner, "Multi-path routing protocols in wireless mobile ad hoc networks: A quantitative comparison", In NEW2AN, 2006.

[14] Marina. M.K, Das. S.R. "On-Demand Multipath Distance Vector Routing in Ad Hoc Networks", Proceedings of the International Conference for Network Protocols, 2001.

[15] Marina. M.K, Das. S.R., “ Ad Hoc On-Demand Multipath Distance Vector Routing", Wireless Communication and Mobile Computing, Vol. 6, Wiley Inter Science, 2006.

[16] C.Perking, E.Belding Royer and S.Das “Ad Hoc On-Demand Distance Vector (AODV) Routing”, RFC-3561, The Internet Engineering Task Force, 2003.

[17] Bo , S M , Xiao , H , Adereti , A , Malcolm , J \& Christianson , B “A Performance Comparison of Wireless Ad Hoc Network Routing Protocols under Security Attack", Proceedings of the Third International Symposium on Information Assurance .

[18] Jaya Jacob and V.Seethalakshmi, "Performance Analysis and Enhancement of Routing Protocol in Manet", International Journal of Modern Engineering Research (IJMER) Vol.2, Issue.2, Mar-Apr 2012.

[19] Vivek B. Kute and M. U. Kharat, "Analysis of Quality of Service for the AOMDV Routing Protocol”, ETASR - Engineering, Technology \& Applied Science Research Vol. 3, No. 1, 2013.

[20] M. K. Marina and S.R.Das, "Ad hoc on-demand multipath distance vector routing”, Wirel. Commun. Mob. Comput., Vol. 6, pp. 969-988, 2006.

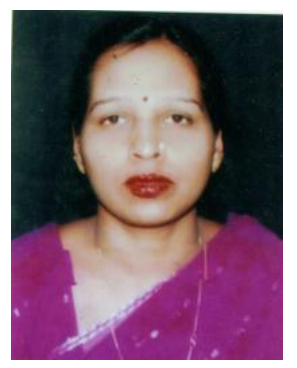

Mrs.Bhawna Mathur, Ph.D scholar, in E.C.E Department, Bhagwant University, Ajmer. M.Tech in Digital Communication from Bhagwant University, Ajmer.

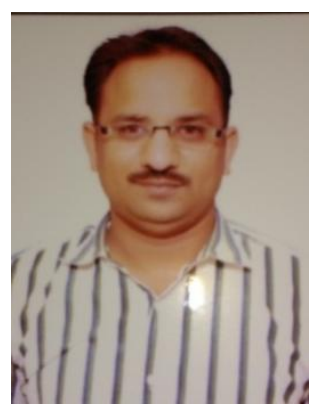

Dr. Anuj Jain . I have contributed 15 years in teaching and administration and research activities of various engineering colleges. As a Head of department, I have more than 17 research papers of electronics, electrical and instrumentation branches. I have helped the management by my dynamic professional work which helped in about critical thinking, ability to solve the problem of research related area like (Manet, DSP, Image Processing) quickly and efficiently. 